In press 2004 - Drug and Alcohol Dependence

\title{
An Internet-based survey method for college student drinking research
}

\author{
Kypros Kypri, $\mathrm{PhD}^{1} *$ \\ Stephen J. Gallagher, $\mathrm{PhD}^{2}$ \\ Martine L. Cashell-Smith, BCom² \\ 1School of Medical Practice and Population Health \\ University of Newcastle \\ Newcastle, Australia \\ ${ }^{2}$ Injury Prevention Research Unit \\ Department of Preventive and Social Medicine \\ University of Otago \\ Dunedin, New Zealand
}

* Author to whom correspondence should be addressed:

School of Medical Practice and Population Health

University of Newcastle

c/o Hunter Population Health

Locked Bag 10, Wallsend 2287 NSW

Australia

E-mail: ～kypros.kypri@hunter.health.nsw.gov.au

Tel: $\quad$ +612 49246205

Fax: $\quad+61249246215$ 
In press 2004 - Drug and Alcohol Dependence

\begin{abstract}
The purpose of this study was to describe and assess the utility of an Internet-based survey method for characterizing the alcohol consumption of college students. After extensive pilot research, a random sample of 1,910 students aged 16-29 years was invited to complete a questionnaire, consisting of a series of web-pages linked to a relational database on a secure website. A branch structure allowed for tailoring of survey items by age and gender. The students received up to nine contacts, including a pre-notice letter with a token gift and an e-mail invitation (Phase 1), a reminder letter and e-mail message (Phase 2), and then telephone reminders and replacement access codes (Phase 3). Noncomputer-users were offered a pen-and-paper alternative, making this a mixed-mode survey. The overall response to the survey was $82 \%(\mathrm{~N}=1,564)$. The median completion time was 16.7 minutes. Participants' comments showed high levels of satisfaction with the survey. Comparison of web $(n=1,501)$ versus pen-and-paper completions $(n=63)$ revealed no modality effects. Technical problems addressed during the course of implementation included web-browser - operating system incompatibilities, and periodic network errors, although these resulted in little lost participation. Internet-based surveys are feasible for college student research and with carefully managed recruitment, can yield a high response.
\end{abstract}

Keywords: college, student, drinking, internet, survey 
"We are experiencing a survey revolution, the consequences of which are yet to be clearly known”

Don Dillman (2000, p.xi).

Surveys continue to be a mainstay of epidemiological research on alcohol. Accordingly, sources of bias affecting survey results warrant continued scientific interest. This is particularly important given recent changes in information technology and its uptake by society. Commentators argue that computers and internet technology (i.e., electronic mail and the World Wide Web) have the potential to bring about significant improvements in survey methods, by reducing various types of survey bias (Dillman, 2000; Schmidt, 1997). The purpose of this paper is to describe and assess the utility of an Internet-based survey method for measuring the alcohol consumption of university students.

The potential for coverage error has been a concern in web surveys, which, until recently, had access to only a small, self-selected portion of the population (Couper, 2000). In New Zealand, upwards of $88 \%$ of university students are assigned an e-mail address and given access to the web (Kypri and McAnally, 2003) similar to access levels for college students in North America (Jones and Madden, 2002). The extent of coverage may therefore be a relatively minor issue, although actual levels of e-mail and web use (distinct from access alone) need to be known. Where these are low, they should be allowed for in survey design and implementation.

In postal, face-to-face and telephone surveys, cost is roughly proportional to the size of the required sample. With Internet surveys, printing, postage, and data entry costs are greatly reduced. Although there can be substantial set-up costs, the marginal cost associated with increasing sample size is comparatively low for Internet surveys 
(Dillman, 2000). Use of the Internet should therefore offer significant cost advantages for large surveys.

Evidence is accumulating on the validity of computerized methods as a means of eliciting information about alcohol and drug use. In a national household survey in which male adolescents were randomly assigned to computer-assisted or pen-and-paper surveys, rates of reporting heavy episodic drinking were higher in the computer-assisted format than in the pen-and-paper format (Turner et al., 1998). A comparison of computer, face-to-face, and pen-and-paper questionnaires for the assessment of alcohol and drug problems among treatment-seeking individuals revealed no important differences in the quality of data, level of disclosure, and reports of consumption, across the three randomly assigned formats (Skinner and Allen, 1983). Although rated as less friendly, the computer assessment took less time, aroused less anxiety, and was rated as more interesting than the other formats (Skinner and Allen, 1983). A study comparing web versus pen-andpaper based assessments of alcohol use and related problems among college students revealed equivalent scores and test-retest reliabilities using the two approaches (Miller et al., 2002).

In a recent editorial, a leading epidemiologist in the alcohol and drug field, stressed the need for further study on survey methods, in particular, on procedures for increasing response rates (Caetano, 2001). Although little research yet exists on procedures for increasing response rates in Internet surveys, postal survey methods have been extensively investigated. In a meta-analytic review of 292 randomized controlled trials of methods designed to increase responses to postal surveys, Edwards et al. (2002) found the following features significantly increased the odds of a response: monetary incentives 
(particularly when not conditional on a response), short questionnaires, personalized contacts, use of colored ink, recorded delivery, first class post, pre-contact notification, follow-up, provision of a second copy of the questionnaire, high levels of interest in the subject of the survey, non-sensitive subject matter, and surveys originating from universities.

Some of these methods can be easily incorporated into a survey design. However, the provision of incentives (conditional or unconditional) and multiple contacts may incur considerable cost, particularly where required sample sizes are large, as is often the case in epidemiological studies. Various factors require consideration. For example, the merit of providing an unconditional token incentive depends on the difference in response rates attained using conditional versus unconditional tokens, the absolute minimum response rate required, and the financial resources available to conduct the survey.

In previous studies we conducted using pen-and-paper surveys for the collection of university student drinking data (Kypri et al., 2002a; Kypri et al., 2002b), missing data levels were as high as $6 \%$ for selected items, and data collection and processing were time and labor intensive. For surveys involving many thousands of individuals (e.g. Wechsler et al., 1994), costs associated with data collection and data processing can be very high. The potential to collect a large volume of data relatively quickly with reduced data processing costs, makes the Internet-based approach highly attractive.

In a pilot study involving 150 students randomly sampled from a university enrolment database, the overall response to a 15-minute Internet-based alcohol survey was $85 \%$ (Kypri and Gallagher, 2003), a substantially higher rate than for surveys with similar 
content in other recent studies of college students (Gliksman et al., 2000; Wechsler et al., 2002). In that study, students were randomly assigned to one of four experimental conditions to examine the efficacy of various types of token incentive. Incentives ranged from a ball-point pen, to a \$5 lunch voucher sent with the invitation to participate (Kypri and Gallagher, 2003). The survey response and latency to respond did not vary significantly by incentive condition. It was concluded from this that Internet surveys are feasible for this university population, and that careful planning of the implementation process is possibly more important than the value of incentives given to participants.

The objectives of this study were to describe and assess the utility of an Internet-based survey of university student alcohol use, and to examine features of the survey process and response in light of evidence-based survey methods.

\section{Method}

Sample

The study sample consisted of 1,910 students aged 16-29 years, randomly selected from the enrolment database of the University of Otago, a public institution, with around 17,000 students, the third largest of eight universities in New Zealand. The south island city of Dunedin, where the main campus is situated, has a population of 120,000 people.

\section{Recruitment}

A three-phase recruitment procedure was used.

Phase 1: Pre-notification and invitation. A personally addressed and signed letter on University of Otago letterhead was mailed to each sampled student (to arrive on day 1 of 
the survey), inviting them to participate in a confidential Alcohol Use Survey via the web. The letter notified recipients that in three days time an e-mail message would be sent to their student e-mail address, and that a hypertext link contained within the message, when clicked, would open their computer's web browser at the site hosting the questionnaire. The letter also contained instructions on how to access the survey by typing in a URL and entering a user code. This was provided for participants who may not have ready access to their student e-mail account. A token gift (a ball point pen valued at US\$0.40) and an information sheet approved by the University of Otago Ethics Committee, that provided details of the survey, were attached to the letter. The e-mail message was generated using mail-merge software so that each message was personally addressed and sent individually rather than as part of a bulk transmission. This message was sent two days after the letter was expected to arrive at the student's in-term residential addresses (day 3), so that they had had the opportunity to read the letter and to see the token gift. Attached to the e-mail message was an electronic copy of the mailed letter and the information sheet describing the research. A hypertext link e-mail address for the researcher, and their postal address and phone number were prominently displayed in the message to allow recipients to make inquiries about the study. In the invitation letter and all subsequent letter and e-mail communication, students were assured that the data they provided were transmitted over a secure connection, remained confidential, and would be used only for the purposes described in the information sheet.

Embedded in the e-mailed hypertext link was a unique identifier (equivalent to a password), which was in turn linked to a separate database containing the sampled individual's identifying information (provided by the university). The hypertext link could be used only once to complete the web questionnaire, i.e., upon submitting the 
final page of the survey, the database was blocked, preventing the student or any other person from over-writing the data on a subsequent occasion. If, however, the student logged out prior to submitting the final page, the hyperlink remained active and they could later re-enter the survey starting from the first page.

Phase 2: Reminders. One week after the first e-mail message was sent (day 10), the survey database was checked to determine if the student had responded. A polite personalized reminder e-mail message was sent to those students who had not yet responded. This also contained a second hypertext link to the web questionnaire in case the previous e-mail message had been deleted. A letter containing the same information was also posted to the student's residential address (day 11).

Phase 3: Intensive follow-up. Ten days after the reminder e-mail message was sent, the survey database was again checked. Students who had not yet responded (and had not indicated refusal to participate) were telephoned (from day 20) to check that they had received the e-mail message and letter and asked if they were willing to participate. Up to five follow-up telephone calls were allowed for in an attempt to make contact. Those who wished to participate but preferred not to use the web-based questionnaire were offered a pen-and-paper alternative. Data collection was completed prior to the commencement of first semester examinations, 56 days after posting of the invitation letter.

\section{Measures}

Participants were asked to use a point-and-click procedure to select their responses to a range of questions concerning: preferred spare-time activities; past alcohol use (age of first full drink, age of first heavy episode, frequency of heavy episodic drinking in last 
year of high school); the Alcohol Use Disorders Identification Test (Saunders et al., 1993); a one-week drinking diary including the quantity and duration of sessions, and the number of times intoxicated; peak consumption in the last 4 weeks; height and weight; positive aspects of drinking; alcohol-related injury; other alcohol-related consequences in last 3 months (22 items); effects of drinking on grades; perceived drinking norms (national, university, and closest friends); secondhand effects of drinking (Langley et al., 2003; Wechsler et al., 1994); drink driving/riding; tobacco use; mental health (Ware and Sherbourne, 1992); and interest in various alcohol-related services (Kypri et al., 2003).

\section{Questionnaire}

The survey instrument consisted of a series of web-pages linked to a relational database. Figure 1 contains an example of one of the web-pages. A demonstration of the entire instrument can be viewed at http://ipru.otago.ac.nz/ausdemo. Responses to most questions were selected using the mouse or arrow keys, from an exhaustive, mutually exclusive set presented in a drop-down menu. Detailed instructions on the use of dropdown menus could be viewed by clicking a button on the first page of questions. For questions in which more than one response option was allowed, checkboxes were used. Instructions were given to place a tick in the checkbox beside as many options as were applicable. For questions that required a free-text response (e.g., a comment) or a numeric response with a range and level of precision that could not be achieved with a drop-down menu (e.g., weight), a textbox was provided. Questions about the number of standard drinks consumed included a reference graphic on the left of the page, depicting common beverages and vessels and the number of standard drinks that each contained. The questionnaire concluded with a thanks page, some space for free-text comments, and hypertext links for help and information about alcohol and health. 
$<$ Figure 1 here $>$

Each page terminated with a "Continue" button and a request to check answers before proceeding. Clicking on the continue button executed Javascript checking routines designed to determine whether a response had been made for each survey item, that freetext responses were of the appropriate type (e.g., numeric versus alphabetical) and were within an acceptable range. Where these routines revealed an omission or an invalid response, an "alert” box was presented in the middle of the screen. This box contained an explanation of the error and a request for the information provided to be corrected. If all checks on a given page were passed, the responses to the items were submitted to the survey database and the next page of questions was presented.

Some items were automatically reworded to reflect the respondent's age and gender. This ensured that questions were phrased according to the appropriate reference group, for example, gender-based definitions of heavy episodic drinking. Similarly, the questionnaire was arranged so that items not relevant to respondents were not presented. For example, respondents who indicated no drinking in the preceding year were not presented with questions concerning their recent alcohol use. The branching and dynamic altering of items were achieved using a combination of Javascript and calculated fields in the survey database, derived from responses submitted earlier in the process of completing the questionnaire.

The questionnaire was accessible via a Secure Socket Layer (SSL) which consists of a third party server with encryption software. Data submitted by respondents are routed via 
the third party server for encryption before being forwarded to host server. This security measure has little noticeable effect on data transmission times. During the survey, the respondent's web browser brings up a dialog box informing the user that their data are being submitted to a secure website. This is seen as an advantage for research purposes. It should, however, be noted that even without SSL, the data submitted consist primarily of a string of digits which are unintelligible without the coding schema which is held by the research team.

\section{Implementation and follow-up procedures}

A relational database, referred to as the Computer Assisted Survey Manager (CASM), was developed to help monitor survey participation and record follow-up contacts. Demographic information (gender, date of birth, ethnicity) and contact details (name, postal addresses, phone numbers, and e-mail address) provided by the University for each sampled participant were stored in CASM and could be used to produce lists suitable for importation into software used for the automatic generation of letters and email messages.

Contact with individual participants was manually recorded in CASM, along with any subsequent action required of the research team. For example, if a reminder telephone call to a participant revealed non-receipt of the letter or e-mail invitations due to a change of address, new contact details could be entered in CASM, with a flag indicating that a new letter and e-mail should be sent. As well as allowing successful contact with the participant to be recorded, a selection of contact types was provided to allow recording of returned e-mails/letters, disconnected or busy telephone lines, wrong numbers, messages 
left on answering machines or with persons other than the participant, and contacts initiated by participants.

\section{Analyses}

Response rates were computed for four groups of sampled individuals: (1) those who completed the entire survey via the web or pen-and-paper, (2) those who fulfilled a core data requirement, i.e., all items up to and including the AUDIT (approximately one quarter of the way through the survey) but not the entire survey, (3) those who provided some data but did not meet the core data requirement, and (4) those who did not provide any data. For the purpose of computing an overall response rate, groups 1 and 2 were classified as respondents and groups 3 and 4 were classified as non-respondents. Survey completion time was computed by subtracting the start time from the finish time for each respondent as recorded on the server.

\section{Assessment of possible modality effects}

For the purpose of examining possible modality effects (i.e., pen-and-paper versus web), we examined a range of demographic and alcohol use variables for pen-and-paper respondents ( $n=63)$, all of whom were sent their forms in Phase 3 of recruitment, with responses attained from web survey respondents. To control for potential effects of time, we compared them with the web-survey respondents who participated in the same phase ( $n=237)$. The comparisons were based on gender, age, age at first full drink, age at first episode of heavy drinking (women $>4$ standard drinks, men $>6$ standard drinks), number of drinking days in the last week, standard drinks consumed in their heaviest episode in the last week, total standard drinks consumed in the last week, and AUDIT score. Chi 
squared tests were used for the categorical variables and t-tests were used for the continuous variables.

Appeal of the survey to students

Free text comments entered by students at the end of the questionnaire were grouped in broad subject categories and reviewed in light of their potential to inform the survey procedures.

\section{Data processing and data quality}

The data were transferred from a Filemaker Pro database to SAS 8.0 (SAS Institute Inc., 1999) for statistical analysis. A data checking and validation procedure was then undertaken. This involved reviewing comments made by participants in which they indicated that an error had been made and then correcting the error in the database. All records were checked for response set, suggestive of indiscriminate responding (e.g., a series of the same responses on the SF-36 sub-scale which contains reverse-coding) and for outliers on particular measures (e.g., Body Mass Index). These records were manually reviewed and excluded if they contained two or more major inconsistencies.

\section{Results}

Survey response

Of 1,910 randomly sampled students 331 (17.3\%) did not respond at all. In eight cases (0.4\%), the sampled individual started the survey but did not proceed far enough to meet the core data requirement. Seven cases $(0.4 \%)$ with evidence of a response set or other significant inconsistencies were considered invalid and were reclassified as nonrespondents. The total non-response was thus $18.1 \%$. Complete responses were received from 1,520 students (79.6\%). In a further 44 cases (2.3\%), although the entire survey was 
not completed, the core data requirement was met. Thus, 1,564 $(1520+44)$ students responded, producing an 81.9\% (95\% CI: 80.1, 83.6) overall response rate.

\section{Reasons for non-participation}

Twenty-four students (1.3\%) refused to participate in the survey: nine were too busy, four were not interested in the subject matter, one for religious reasons, one cited a policy of not participating in surveys, and nine gave no explanation. A further 224 students (11.7\%) requested, and were supplied with, either a paper copy of the questionnaire, a new hypertext link, or were reminded about the survey, but for unknown reasons did not start the questionnaire. Finally, 77 students (4.0\%) could not be contacted at all.

\section{Completion time}

The median time required to complete the web questionnaire was 16.7 minutes (females: 16.1 minutes, males: 17.5 minutes). Males took significantly longer to complete the survey than did females (SAS Institute Inc., 1999). The fastest $25 \%$ of the respondents completed the questionnaire in less than 13.5 minutes, while the slowest $25 \%$ took more than 20.5 minutes. All but $5 \%$ of the sample completed the questionnaire in less than 30 minutes.

\section{Temporal pattern of response}

Figure 2 shows the number of questionnaire completions by day of survey implementation. The point at which each phase of recruitment and follow-up commenced is indicated. The sharp peak in responses reflects the posting of the invitation letter and email message. Of the eligible sample, 828 (43.4\%) responded during Phase 1 of recruitment, i.e., before the reminder e-mail message and letter were sent. The reminder 
e-mail message and letter (Phase 2) generated a smaller peak in the response profile, with 436 individuals (22.8\% of the eligible sample) responding before intensive follow-up began. By the end of the extensive follow-up period (Phase 3), a further 300 individuals (15.7\%) had responded.

$<$ Figure 2>

\section{Modality effects}

Table 1 shows that participants who completed the survey by pen-and-paper, did not differ significantly from those who completed the web survey, by gender, age, and a range of alcohol use variables.

$<$ Table $1>$

\section{Technological problems}

A seemingly inexplicable problem was encountered by participants using Netscape 4.5 on Macintosh computers connected to the University network. Despite having tested this configuration before commencement of the survey, when a particular web-page was submitted (the drinking diary), Netscape ceased functioning. The problem was resolved by removing a border around the table containing the drinking diary responses, but no explanation for this problem could be found.

A similar problem was encountered by two users of older web browsers. For these respondents the JavaScript checking routines were not accurately interpreted, preventing advancement to the next page. These problems illustrate the difficulties associated with 
undertaking surveys on the Internet, with some of the large number of browser and operating-system combinations leading to unpredictable results.

No server errors were encountered during data collection. Soon after the survey was completed, however, a change in the University’s network resulted in the server hosting the survey being shut down for several days. Had this occurred in the first week of the survey, it would have been a significant barrier to survey participation and may therefore have reduced the response rate.

Students' views of survey procedures

Free text comments entered by respondents provided data which was of assistance in evaluating the survey methods. Of 448 free text comments entered, 209 referred primarily to the survey procedures. These fell into seven categories: (i) comments concerning the length, principally that the survey was too long ( $n=23$ ); (ii) queries or suggestions ( $\mathrm{n}=5$ ); (iii) comments about the structure of the survey or wording of questions (n=37); (iv) comments about the difficulty of estimating drinking norms ( $n=12)$; (v) corrections of responses given during the survey ( $n=13$ ); (vi) suggestions for other items to include, (n=33) e.g., about other drug use; and (vii) compliments/well wishes $(n=85)$.

The value of incentives and multiple modes of contact were remarked upon in a number of comments. For example:

Can I just say you guys did the right thing to get me to fill out that really long survey! The free pen got my attention (students love free stuff by the way). Then I 
forgot about it till I got a second letter, and I thought, alright I'll do it (18 year-old female).

The free pen was really good, for me and most of my friends it made us decide to definitely do the survey, I would probably feel guilty about accepting the pen otherwise (21 year-old male).

Most comments suggested that students found the web-based approach acceptable. For example:

I found the survey interesting to answer and would love to be informed of the results when the project is completed (20 year-old female).

I think this sort of anonymous web based survey is a great idea and that researchers within the university should use it more (22 year-old female).

This respondent may have meant “confidential” rather than "anonymous”. Alternatively, her choice of word suggests that the manner in which the survey was conducted made her feel comfortable to respond honestly.

\section{Discussion}

We observed a spike in the response rate in the three days following posting of the invitation letter and e-mail message, followed by a refractory period. The posting of a second letter and reminder e-mail message resulted in another spike in the response rate, followed by a rapid decline. Intensive follow-up, particularly with the use of telephone reminders and the provision of replacement e-mails containing the hypertext link to the 
web questionnaire, produced a steady response for the following four weeks. This response profile is consistent with findings from randomized controlled trials of postal survey methods (Edwards et al., 2002), which have shown that multiple contact attempts, and the provision of replacement forms (in this case replacement hypertext links), are important methods of attracting survey participation.

The response rate achieved in this full implementation of the Alcohol Use Survey (82\%) fell within the $95 \%$ confidence interval of the rate estimated in a pilot study: $85 \%$ (79\%, 91\%), using a similar implementation procedure (Kypri and Gallagher, 2003). These rates compare favorably with those reported for other surveys of college student alcohol use. In the two largest (pen-and-paper) surveys of college student drinking in recent years - conducted in the USA and Canada - the response rates were 52\% (Wechsler et al., 2002) and 51\% (Gliksman et al., 2000).

Notably, response rates reported by Wechsler and colleagues for the ongoing College Alcohol Survey, have declined precipitously from the 70\% achieved in the first survey, conducted in 1993 (Wechsler et al., 1994). While it is possible that non-respondents do not differ in their alcohol consumption from those who respond, the potential for nonresponse error increases as the response rate falls (Caetano, 2001).

In a study in which American college students were randomly assigned to postal versus web-based survey conditions, response rates were 40\% and 63\% (McCabe et al., 2002). There are some differences between the present study and that by McCabe et al., including: time in the field (56 days versus one month); modes of contact (multiple versus single); and incentives (a pen given unconditionally versus a book voucher given conditionally). 
In all of the studies discussed above, conditional token incentives were offered: entry to a cash prize draw (Gliksman et al., 2000; Wechsler et al., 2002) or a book voucher (McCabe et al., 2002). It is possible that New Zealand university students are more willing to participate in surveys than their North American counterparts, although response rates for national household surveys of alcohol use in New Zealand (Casswell et al., 2002) fall within the $60 \%-80 \%$ range attained in other developed countries, including the USA and Canada (World Health Organization, 2000). There may be value in conducting a study using a standard web-based method in public universities of similar size in several different countries.

It may seem ironic that potential participants received a pen and a conventionally mailed invitation to complete a web questionnaire. Although this study does not demonstrate the independent effects of these measures, the findings of controlled trials suggest that the use of both a token gift and a pre-notice letter increase the odds of participation (Edwards et al. 2002). In a discussion of the development of Internet survey methods, Dillman (2000) speculates on how incentives might be provided for Internet survey participation. In the present study, the mailing of a pre-notice letter offered an opportunity to include a token gift. The pen and the pre-notice were positively remarked upon by several participants.

In $3 \%$ of cases participation appears to have been facilitated by providing a pen-andpaper version of the survey. Comments made to members of the research team conducting the follow-up suggested that these individuals would not have participated without this option. Importantly, those who completed a pen-and-paper questionnaire did not differ significantly in their alcohol use from the remainder of the sample. This is 
consistent with the results obtained by McCabe et al. (2002), in which students randomized to a web-survey condition did not differ significantly in their alcohol use from those randomized to a pen-and-paper condition. As was observed in the McCabe et al. study, there were missing data for selected items in the paper forms, while missing data in the web-completed questionnaires were confined to cases where participants stopped part way through the survey.

As survey response rates have fallen over the past two decades (Caetano, 2001), methodologists have recommended increasing the maximum number of contacts from around five (Dillman, 1978) to around nine (Dillman, 2000). In this study, the response rate after the first two contacts (Phase 1) was 43\%, after the third and fourth contacts (Phase 2) it was 66\%. From the point at which intensive follow-up commenced (Phase 3), a further $16 \%$ of the sample submitted data. It is likely that some Phase 3 responses would have occurred without the reminder phone calls, such that we can attribute at least $80 \%$ of the responses ( $66 \%$ / $82 \%)$ to the first four contacts: two by letter and two by email. There would be value in knowing the yield of the additional effort required to elicit the final $20 \%$ of responses.

In a separate study assessing non-response error we compared the alcohol use levels of Phase 3 versus Phase 1 and 2 respondents, finding that the Phase 3, or 'late', respondents, had slightly higher AUDIT scores and experienced more alcohol-related problems than Phase 1 and 2 respondents, but that this had only small effects on estimates of either alcohol consumption or the prevalence of hazardous drinking (Kypri et al., 2004). It was concluded that the yield of Phase 3 did not warrant the intensive effort, and that for future surveys in this population it would be preferable to increase the initial sample size and to 
include additional e-mail or letter contacts with a view to achieving a response rate of 70 80\% (Kypri et al., 2004).

In their meta-analytic review of methods to increase survey participation, Edwards et al. (2002) found that survey response was inversely associated with questionnaire length. Over one quarter of the sample in this study took more than 20 minutes to complete the web questionnaire. Some comments received from participants indicated that this was too long. There were, however, only 52 respondents (3\%) who started but did not complete the survey, and only eight of these failed to meet the core data requirement. Use of separate web-pages rather than a continuous scroll-down page increased download times but was considered necessary for a number of reasons. Personalization of the survey by gender, age, and drinking status required data on these variables to be submitted and processed to update fields in subsequent questions. Also, the survey branch structure, in which respondents saw only questions relevant to them, required that data be submitted at various points, making a series of pages a technical necessity. Another advantage of using multiple pages is that if there is a computer crash, network error, phone disconnection, or the participant decides to stop part way through the survey, not all of the data are lost. As the bandwidth of network and dial-up connections is increased, download times will decrease, which will reduce the time required to complete web questionnaires.

A growing body of research on Internet survey methods may yield benefits for intervention research aimed at reducing hazardous drinking via the provision of personalized feedback. A number of studies, including one based on the sample used in this study (Kypri and Langley, 2003), show that college students tend to over-estimate 
their peers' drinking levels. If this tendency to over-estimate is a causal factor in the individual’s drinking, as has been suggested (Perkins, 2002), providing personalized feedback in which normative misperceptions are corrected, may reduce heavy episodic drinking (Saunders et al., 2004).

\section{Conclusions}

For the survey researcher, the Internet offers many exciting possibilities. In the general population, in which Internet access is still well below levels required to overcome problems of coverage bias, many of these possibilities cannot yet be realized. In certain groups, however, coverage is presently sufficient for the use of this technology for both epidemiological purposes and health promotion (e.g., college and high school students).

Research is beginning to mount on various aspects of Internet technology as they apply to survey research. This paper has described an evidence-based approach which yielded an $82 \%$ response with high data quality. Survey modality effects appeared to be minor and to favor a computerized approach, consistent with the results of other key studies showing higher levels of participation (McCabe et al., 2002) and greater reporting of sometimes stigmatized behaviors (Turner et al., 1998). Research is required on some elements of survey design and their effects on measurement accuracy. Further research is also required on possible non-response biases.

\section{Acknowledgements}

This research was funded by the Health Research Council of New Zealand and the Alcohol Advisory Council of New Zealand. The authors are grateful to Associate Professor David Chalmers and two anonymous reviewers for valuable comments on an earlier draft of the paper. 


\section{References}

Caetano, R. (2001) Non-response in alcohol and drug surveys: a research topic in need of further attention. Addiction 96, 1541-5.

Casswell, S., Huckle, T. and Pledger, M. (2002) Survey data need not underestimate alcohol consumption. Alcohol Clin Exp Res 26, 1561-7.

Couper, M. (2000) Web surveys: a review of issues and approaches. Public Opin Q 64, 464-94.

Dillman, D. A. (1978) Mail and telephone surveys : the total design method. Wiley, New York.

Dillman, D. A. (2000) Mail and internet surveys : the tailored design method. J. Wiley, New York.

Edwards, P., Roberts, I., Clarke, M., DiGuiseppi, C., Pratap, S., Wentz, R. and Kwan, I. (2002) Increasing response rates to postal questionnaires: Systematic review. Br Med J 324, 1183.

Gliksman, L., Demers, A., Adlaf, E. M., Newton-Taylor, B. and Schmidt, K. (2000) Canadian Campus Survey 1998. Centre for Addiction and Mental Health, Toronto, Canada.

Jones, S. and Madden, M. (2002) The Internet goes to college: How students are living in the future with today's technology. Pew Internet and American Life Project. http:/ / www.pewinternet.org.

Kypri, K. and Gallagher, S. J. (2003) Incentives to increase participation in an internet survey of alcohol use: a controlled experiment. Alcohol Alcohol 38, 437-441. 
Kypri, K. and Langley, J. D. (2003) Perceived norms and their relation to university student drinking. J Stud Alcohol 64, 829-834.

Kypri, K., Langley, J. D., McGee, R., Saunders, J. B. and Williams, S. (2002a) High prevalence, persistent hazardous drinking in New Zealand tertiary students. Alcohol Alcohol 37, 457-64.

Kypri, K. and McAnally, H. (2003) Tertiary student access to the Internet in New Zealand: Implications for health research. Occasional Report. Dunedin: Injury Prevention Research Unit, Dunedin.

Kypri, K., McGee, R., Saunders, J. B., Langley, J. D. and Dean, J. I. (2002b) Interpretation of items on the AUDIT questionnaire. Alcohol Alcohol 37, 465-7.

Kypri, K., Saunders, J. B. and Gallagher, S. J. (2003) Acceptability of various brief intervention approaches for hazardous drinking among university students. Alcohol Alcohol 38, 626-628.

Kypri, K., Stephenson, S. C. R. and Langley, J. D. (2004) Assessment of nonresponse error in an Internet survey of university student alcohol use. Alcohol Clin Exp Res 28 (4).

Langley, J. D., Kypri, K. and Stephenson, S. (2003) Secondhand effects of alcohol use among university students: Computerised survey. Br Med J 327, 10234.

McCabe, S. E., Boyd, C. J., Couper, M. P., Crawford, S. and D'Arcy, H. (2002) Mode effects for collecting alcohol and other drug use data: Web and U.S. mail. J Stud Alcohol 63, 755-61. 
Miller, E. T., Neal, D. J., Roberts, L. J., Baer, J. S., Cressler, S. O., Metrik, J. and Marlatt, G. A. (2002) Test-retest reliability of alcohol measures: Is there a difference between Internet-based assessment and traditional methods? Psychol Addict Behav 16, 56-63.

Perkins, H. W. (2002) Social norms and the prevention of alcohol misuse in collegiate contexts. J Stud Alcohol Suppl 14, 164-172.

SAS Institute Inc. (1999) SAS/STAT User's Guide, Version 8.

Saunders, J. B., Aasland, O. G., Babor, T. F., de la Fuente, J. R. and Grant, M. (1993) Development of the Alcohol Use Disorders Identification Test (AUDIT): World Health Organisation Collaborative Project on Early Detection of Persons with Harmful Alcohol Consumption--II. Addiction 88, 791-804.

Saunders, J. B., Kypri, K., Walters, S., Laforge, R. G. and Larimer, M. E. (2004) Approaches to brief intervention for hazardous drinking in young people. Alcohol Clin Exp Res 28, 332-9.

Schmidt, W. C. (1997) World-Wide Web survey research: Benefits, potential problems, and solutions. Behav Res Methods, Instrum Comput 29, 274-279.

Skinner, H. A. and Allen, B. A. (1983) Does the computer make a difference? Computerized versus face-to-face versus self-report assessment of alcohol, drug, and tobacco use. J Consult Clin Psychol 51, 267-275.

Turner, C. F., Ku, L., Rogers, S. M., Lindberg, L. D., Pleck, J. H. and Sonenstein, F. L. (1998) Adolescent sexual behaviour, drug use, and violence: Increased reporting with computer survey technology. Science 280, 867-873. 
Ware, J. E., Jr. and Sherbourne, C. D. (1992) The MOS 36-item short-form health survey (SF-36). I. Conceptual framework and item selection. Med Care 30, $473-83$.

Watson, P. D., Denny, S. J., Adair, V., Ameratunga, S. N., Clark, T. C., Crengle, S. M., Dixon, R. S., Fa'asisila, M., Merry, S. N., Robinson, E. M. and Sporle, A. A. (2001) Adolescents' perceptions of a health survey using multimedia computer- assisted self-administered interview. Aust NZ J Public Health $25,520-4$

Wechsler, H., Davenport, A., Dowdall, G., Moeykens, B. and Castillo, S. (1994) Health and behavioral consequences of binge drinking in college. A national survey of students at 140 campuses. JAMA 272, 1672-7.

Wechsler, H., Lee, J. E., Kuo, M., Seibring, M., Nelson, T. F. and Lee, H. (2002) Trends in college binge drinking during a period of increased prevention efforts. Findings from four Harvard School of Public Health College Alcohol Study surveys: 1993-2001. J Am Coll Health 50, 203-17.

World Health Organization (2000) International Guide for Monitoring Alcohol Conumption and Related Harm. Department of Mental Health and Substance Dependence, World Health Organisation, Geneva. 
Table 1. Demographic details and alcohol use measures for respondents using paper versus web questionnaires

\begin{tabular}{lcccc}
\hline & \multicolumn{4}{c}{ Questionnaire modality } \\
Measure & Paper & Web & $t / \chi^{2}$ & $p$ \\
& $(\mathrm{n}=63)$ & $(\mathrm{n}=237)$ & statistic & value \\
\hline Gender of respondent (\% women) & $54.8 \%$ & $54.4 \%$ & 0.00 & 0.95 \\
Age of respondent (in years) & 20.8 & 20.6 & 0.66 & 0.51 \\
Age at first full drink (drinkers only: 62 paper, 229 web) & 13.3 & 13.5 & 0.80 & 0.43 \\
Age at first episode of binge drinking* (women >4 standard & & & & \\
drinks, men >6 standard drinks*) & 14.8 & 15.1 & 1.55 & 0.12 \\
Number of drinking days in last week & 1.0 & 1.3 & 1.56 & 0.12 \\
Standard drinks* consumed in heaviest episode in last week & 5.6 & 6.1 & 0.49 & 0.63 \\
Standard drinks consumed in last week & 7.7 & 9.4 & 0.89 & 0.37 \\
AUDIT score & 11.4 & 11.4 & 0.08 & 0.94 \\
\hline
\end{tabular}

Note: With the exception of gender, statistics are arithmetic means.

* Excludes 29 individuals who reported never binge drinking (3 paper, 26 web)

**1 standard drink $=10$ grams of ethanol 
Figure 1. Sample page from the web-based questionnaire

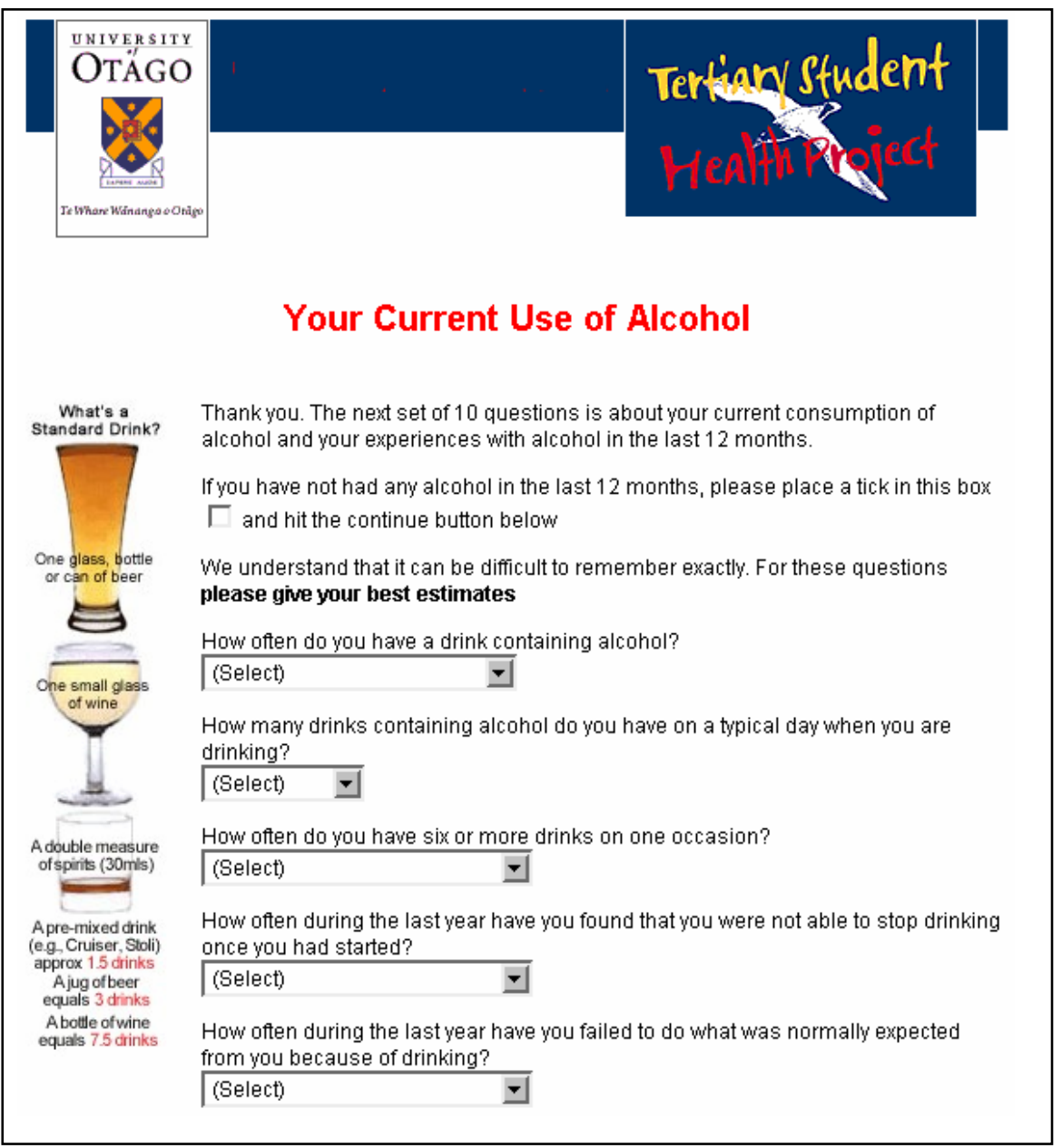


Figure 2. Temporal profile of survey responses
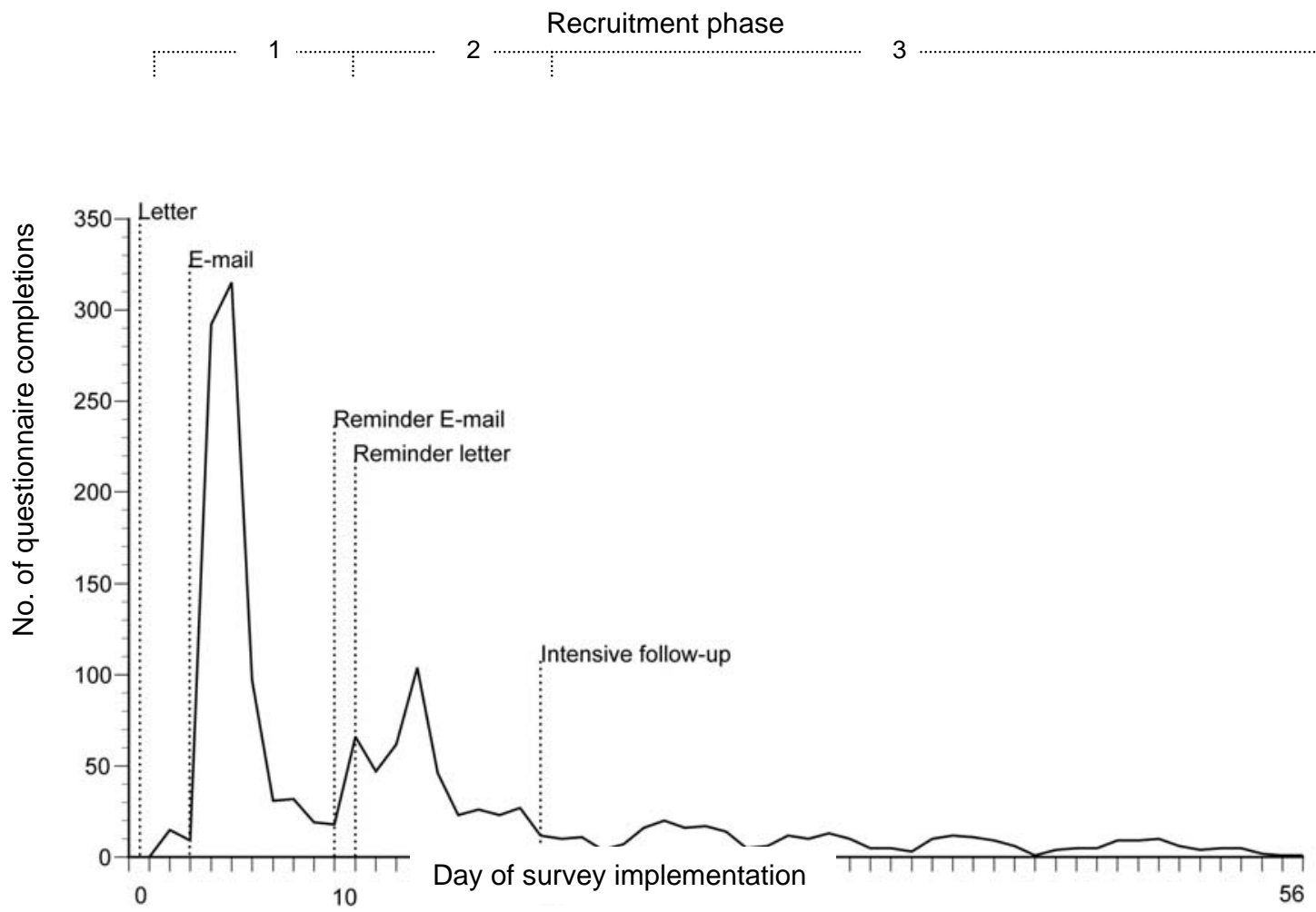